EBM - Verdict

\title{
Is it time to end general health checks?
}

\section{Carl Heneghan, Kamal R. Mahtani.}

General Health checks are used in several countries. However, the evidence suggests they are not effective and offer low-value healthcare.

EBM Verdict on: General health checks in adults for reducing morbidity and mortality from disease. Krogsbøll LT, Jørgensen KJ, Gøtzsche PC. Cochrane Database Syst Rev. 2019 Jan 31;1:CD009009. doi: 10.1002/14651858.CD009009.pub3.

In some countries, general health checks form part of routine health care. In the UK, for instance, an NHS health check is offered to everyone over the age of 40 and under 74. In America, about one in five adults (approximately 64 million Americans) have an annual general examination, costing more than $\$ 5$ billion. [1] However, it is not clear whether health checks are effective for improving morbidity, quality of life or mortality.

A recent update of a Cochrane review aimed to quantify the benefits and harms of health checks (defined as 'screening for more than one disease or risk factor in more than one organ system') in healthy adults compared with no checks. [2]

The review included 17 randomised trials, of which 15 reported outcome data for 251,891 participants. Nine of the trials were set in a medical centre, five in general practice and one in a workplace setting. Overall health checks did not affect total mortality, risk ratio (RR) 1.00 (95\% confidence interval (CI) 0.97 to 1.03); or cancer mortality, RR 1.01, (95\% CI 0.92 to 1.12). Health checks also had no significant effect on cardiovascular mortality, fatal and non-fatal ischaemic heart disease and non-fatal stroke.

The risk of bias across these outcomes was considered generally low. However, there were issues with lack of blinding and missing outcome data as well as contamination in the control groups. Interpretation of the review findings are further limited by problems with generalisability: six trials were done fifty years ago - in the 1960s - an era when modern preventive drug therapy was not part of routine practice. There is also no accepted consensus on the definition and main components of health checks, [3] and as a consequence, the studies differed significantly in the components of their checks.

In the last 20 years, little new evidence for general health checks has emerged, and there have been no high-quality evidence comparing health checks to other forms of health promotion and prevention. The reasons for the lack of forthcoming evidence is not clear. Much of the recent focus has evaluated risk stratification and preventive drug treatments. However, the evidence for risk assessment in the primary prevention of cardiovascular disease is poor in quality, and evidence from systematic reviews shows no effect of risk assessment on reducing CVD morbidity or mortality. [4] Results for primary preventive treatments can also be disappointing. For example, the CVD benefits of aspirin, are outweighed by the risk of bleeding, and it is, 
therefore, not be offered for primary CVD prevention to otherwise well elderly patients. [5] Similarly, statins in participants over 75 without vascular disease, do not appear to reduce major vascular events. [6]

Primary care is frequently the environment of choice to implement health checks. However, systematic evidence (similar to the review described above) does not appear to support this choice. A previous 2014 systematic review of primary care-based health checks including six studies, found that while health checks were associated with small improvements in surrogate outcomes, they did not lead to reductions in morbidity and had no effect on mortality. [7] A more recent controlled study of 38 General Practices in the UK providing NHS Health Checks found that compared to 41 control practices providing usual care there was no difference in the reported prevalence of cardiovascular disease including diabetes and hypertension over three years. [8] NHS Health Checks are unlikely to be cost-effective, as a recent modelling study reported targeted policies such as reducing unhealthy diets and smoking were more likely to be cost-effective. [9]

The results of this recent systematic review and evaluation of health checks in general practice challenge the assumption that the detection of disease is a proxy for better healthcare - health policy frequently suggests otherwise.

Forty years ago, Haynes and Sackett first showed that detection of hypertension in an industrial setting led to those labelled as hypertensive having more time off from work. [10] Health checks can expose patients to unnecessary tests and interventions as well as the problems of overdiagnosis. The focus on testing in general health checks can also prove costly. As an example, US spending on health checks equals all expenditure on breast cancer care. [11] It is, therefore, essential that the research community prioritise addressing the uncertainties in the evidence base for the value of health checks and that health policymakers consider the knowledge gaps when considering their routine use.

\section{EBM Verdict:}

There is no convincing evidence to support the implementation of general health checks in primary care. They appear not to affect clinically important outcomes, and there is no highquality evidence to suggest they are cost-effective, particularly when compared to routine primary care.

\section{Competing interests}

CH and KM are supported by the NIHR School for Primary Care Research Evidence Synthesis Working group (NIHR SPCR ESWG project 390). CH is also supported by the NIHR Oxford Biomedical Research Centre, is an NIHR Senior Investigator and Editor in Chief of BMJ Evidence-Based Medicine. KM is an Associate Editor of BMJ EBM.

\section{REFERENCES}

1 Mehrotra A, Zaslavsky AM, Ayanian JZ. Preventive health examinations and preventive gynecological examinations in the United States. Arch Intern Med 2007;167:1876-83.

2 Krogsbøll LT, Jørgensen KJ, Gøtzsche PC. General health checks in adults for reducing morbidity and mortality from disease. Cochrane Database Syst Rev 2019;1:CD009009.

3 López-García-Franco A E al. Health checks in general practice: evidence first, not last. PubMed - NCBI. https://www.ncbi.nlm.nih.gov/pubmed/26895552 (accessed 19 Jun 2019). 
4 Collins DRJ, Tompson AC, Onakpoya IJ, et al. Global cardiovascular risk assessment in the primary prevention of cardiovascular disease in adults: systematic review of systematic reviews. BMJ Open 2017;7:e13650.

5 O'Sullivan JW. Aspirin for the primary prevention of cardiovascular disease in the elderly. BMJ Evidence-Based Medicine 2019;:bmjebm - 2018-111138.

6 Heneghan C, Mahtani KR. Absolute effects of statins in the elderly. BMJ Evidence-Based Medicine 2019;:bmjebm - 2019-111189.

$7 \quad$ Si S E al. Effectiveness of general practice-based health checks: a systematic review and meta-analysis. - PubMed - NCBI. https://www.ncbi.nlm.nih.gov/pubmed/24567582 (accessed 19 Jun 2019).

8 Caley M E al. The impact of NHS Health Checks on the prevalence of disease in general practices: a controlled study. - PubMed - NCBI. https://www.ncbi.nlm.nih.gov/pubmed/25071065 (accessed 19 Jun 2019).

9 Kypridemos C, Collins B, McHale P, et al. Future cost-effectiveness and equity of the NHS Health Check cardiovascular disease prevention programme: Microsimulation modelling using data from Liverpool, UK. PLoS Med 2018;15:e1002573.

10 Haynes RB, Sackett DL, Taylor DW, et al. Increased absenteeism from work after detection and labeling of hypertensive patients. $N$ Engl J Med 1978;299:741-4.

11 Tanne JH. Annual check-ups aren’t needed, US study says. BMJ : British Medical Journal 2007;335:631. 